\title{
Determinantes socioeconómicos y emocionales en el consumo de nuevos alimentos. Un estudio piloto
}

\author{
R. Barrena ${ }^{1, *}$, T. García ${ }^{1}$ y N. López-Mosquera² \\ 1 Dpto. Gestión de Empresas, Universidad Pública de Navarra, Campus de Arrosadía, 31006 Pamplona, \\ España \\ 2 Facultad de Estudios Empresariales y Turismo, Universidad de Extremadura, 10071 Cáceres, España
}

\section{Resumen}

A pesar de los esfuerzos realizados por las empresas, el índice de fracaso de los nuevos productos es muy elevado, debido fundamentalmente a una falta de entendimiento del consumidor. De ahí que el éxito de un nuevo producto alimentario en el mercado se base en una mejor orientación al mercado. Tradicionalmente se ha relacionado la adopción de nuevos productos con las características socioeconómicas de los consumidores, sin embargo trabajos más recientes han mostrado la importancia de la innovativeness o tendencia innovadora del consumidor como un factor positivo en la adopción de innovaciones y aún más, la aceptación de nuevos productos se relaciona con las emociones percibidas en el momento de consumo.

El objetivo de este trabajo es analizar en qué medida el carácter innovador del consumidor determina el consumo de nuevos alimentos, así como en qué medida las emociones ayudan a mejorar la predicción del consumo de nuevos alimentos. Se han utilizado modelos de ecuaciones estructurales para comprobar las relaciones causales, llegando a concluir que el nivel educativo y la percepción de emociones positivas influyen positivamente en el carácter innovador de los consumidores y que éste determina significativamente la frecuencia de consumo de cápsulas de café.

Palabras clave: Carácter innovador, emociones, modelos de ecuaciones estructurales, nuevos alimentos.

\section{Abstract \\ Socio-economic and emotional determinants in the consumption of new food. A pilot study}

Despite the efforts made by the firms, food innovations have a high failure rate, owing to a fault of understanding of the consumer. Hence, the success of the new alimentary product in the market is based on a better orientation to the market. Traditionally, the adoption of new products has been related to the socioeconomic characteristics of the consumers, however more recent works have shown the importance of "innovativeness" or the consumer's innovative tendency like a positive factor the adoption of the innovations and, even more, the acceptation of the new products is related with the emotions felt in the moment of consumption.

The aim of this paper is analyze in which way the consumers' innovative tendency determine the consumption of the new aliments, as well as in which way the emotions help improving the prediction of the new aliments consume. Structural Equation Models have been used to check the casual relations,

\footnotetext{
* Autor para correspondencia: ramo.barrena@unavarra.es http://dx.doi.org/10.12706/itea.2016.006
} 
coming to the conclusion that the educative level and the perception of positive emotions influence positively in the innovator character of the consumers and that determines significantly the frequency of the coffee capsules consume.

Key words: Innovativeness, emotions, structural equation models, novel foods.

\section{Introducción}

La globalización e internacionalización de los mercados, la concentración de la oferta como resultado de la acción de grandes empresas tanto en distribución como en producción, y la existencia de un consumidor cada vez más exigente y consciente de lo que busca en un producto alimenticio ha originado una competitividad creciente en el sector alimentario. Este nuevo entorno competitivo ha provocado que las empresas se hayan visto en la necesidad de innovar y desarrollar nuevos productos alimentarios para satisfacer las necesidades y deseos de los mercados y mejorar la posición competitiva de las empresas (Baregheh et al., 2009; Naidoo, 2010). Las innovaciones han contribuido significativamente a mejorar la calidad de vida de los consumidores, proporcionándoles beneficios tanto individualmente, como a la sociedad en una escala más amplia. Muchas de estas innovaciones se han incorporado a la vida cotidiana con un buen nivel de aceptación por parte del consumidor, mientras que otras innovaciones han encontrado resistencia. Dentro del sector alimentario se encuentra la misma situación, con algunas innovaciones fácilmente adoptadas y otras rechazadas por los consumidores. En este sector el éxito de lanzamiento de nuevos productos es relativamente bajo, con índices de fracaso entorno al 40-90\% (Gourville, 2006; Gresham et al., 2006).

Este alto ratio de fracaso es motivado en muchas ocasiones por una falta de entendimiento del consumidor y una falta de orientación al mercado de las empresas. El consumidor de hoy en día es cada vez más variable y menos predecible debido a los cambios significativos en los estilos de vida, a los cambios demográficos, intercambios culturales y al alto nivel de comunicación (Capitanio et al., 2009; Fortuin y Omta, 2009; Kühne et al., 2010). Por ello, tener un conocimiento mejor de lo que los consumidores quieren, de cómo cambian sus necesidades y cómo estos cambios pueden ser abordados inmediatamente, es decir, orientarse al mercado, se convierte no solo en un factor de éxito para las empresas agroalimentarias, sino también en un factor de supervivencia (Costa et al., 2004). El éxito de las innovaciones se basa en entender al consumidor, qué características y beneficios busca en los productos que adquiere y consume, y que aspectos de su propia personalidad está proyectando a través de estos productos.

La investigación centrada en el consumidor ha considerado las características mentales, de comportamiento y demográficas asociadas con la disposición a adoptar innovaciones. Muchos trabajos han relacionado la adopción de nuevos productos con las características socioeconómicas de los consumidores (Michon et al., 2010). Ahora bien, la mayor parte de los estudios indican que las características personales afectan a la adopción de innovaciones de forma débil (Urala y Lähteenmäki, 2007; Bellows et al., 2010). Parece ser que un predictor más consistente con el comportamiento innovador es la disposición a probar nuevos productos dentro de una categoría de productos. Distintos trabajos han mostrado el papel de la tendencia innovadora del consumidor o innovativeness, con un impacto positivo en la adopción de innovaciones (Banterle et al., 2011; Dobre et al., 2009; Goldsmith y Flynn, 1995; Roehrich, 2004). 
Más recientemente, la atención se ha puesto en aspectos de naturaleza más psicológica, como son las emociones percibidas en el momento de consumo, las cuales juegan un papel adicional en la explicación del comportamiento del consumidor en relación con los alimentos. El importante papel que las emociones juegan en la vida de los consumidores sugiere que éstas pueden explicar el comportamiento en situaciones donde otros aspectos como las actitudes no pueden explicar la variabilidad del comportamiento (Laverie et al., 1993). Kulviwat et al. (2007) muestran que incluir emociones en un modelo de aceptación de nuevos productos mejora ampliamente la adopción de estos. Este estudio piloto pretende avanzar un paso más en este último enfoque, ya que trata de determinar en qué medida aspectos personales del consumidor influyen en la elección de nuevos alimentos. Se trata de pasar de la perspectiva tradicional (Lancaster, 1996) que consideraba únicamente las características físicas del producto, hacia una perspectiva más amplia que analice la influencia de los aspectos personales del elector. Este planteamiento teórico encaja en un entorno donde los consumidores tienen más deseos que necesidades, lo que implica que busquen funciones adicionales que den valor añadido al producto. El conocimiento de esta información permitirá a los productores mejorar sus estrategias productivas y comerciales.

A partir de lo anteriormente indicado, el objetivo de este estudio piloto es analizar en qué medida el carácter innovador del consumidor determina el consumo de nuevos alimentos, y como las emociones contribuyen a mejorar la predicción del consumo de nuevos alimentos.

Para ello se propone una comparación de dos modelos: un modelo inicial en el que se considera el carácter innovador del consumidor y un modelo ampliado donde se incorporan las emociones percibidas por parte del consumidor al consumir un nuevo alimento.
De esta forma se identificará el efecto que tienen las emociones sobre la adquisición de nuevos alimentos.

\section{Material y métodos}

Planteamiento teórico

Tendencia innovadora del consumidor (innovativeness)

El concepto de tendencia innovadora o innovativeness en el consumidor ha sido de gran interés para la literatura durante décadas y ha sido ampliamente desarrollado en los últimos años (Roehrich, 2004). Las primeras nociones de este concepto fueron introducidas por Rogers (1962), quien definió la innovativeness como el "grado por el que un individuos es relativamente pionero en la adopción de una nueva idea con respecto a otros miembros del sistema". Sin embargo, no hay consenso con respecto al significando de carácter innovador. Puede ser descrito como una compra temprana de un nuevo producto, así como la tendencia de ser atraído por nuevos productos (Steenkamp et al., 1999). Hirschman (1980) y Manning et al. (1995) definieron este carácter innovador como el deseo inherente de búsqueda de novedades y creatividad. Steenkamp et al. (1999) vieron el carácter innovador como la predisposición a comprar productos y marcas nuevas y diferentes más que permanecer en las elecciones previas y en los patrones de consumo habituales.

La mayor parte de la literatura en relación con la innovativeness del consumidor se encuentra en la literatura de la psicología del consumidor y del marketing. Esta investigación se ha centrado en el concepto de innovativeness como un aspecto de la personalidad (Hirschman, 1980; Manning et al., 1995; Steenkamp et al., 1999; Venkatraman y Price, 1990). El objetivo era identificar a los consumidores innovadores 
basándose en su predisposición hacia los productos innovadores, como la predisposición a un comportamiento de adopción innovador (Foxall, 1988; Foxall, 1995; Goldsmith et al., 1998; Manning et al., 1995; Michon et al., 2010). Los consumidores con un mayor carácter innovador pueden ser caracterizados por variables como la renta, edad, educación, participación social, predisposición a asumir riesgos (Gatignon y Robertson, 1991; Steenkamp et al., 1999) y mayor opinión de liderazgo (Gatignon y Robertson, 1991).

La mayor parte de la literatura señala que la edad tiene un efecto negativo en el carácter innovador del consumidor. Tradicionalmente los consumidores más jóvenes son más innovadores (Rogers, 2003) y los consumidores de más edad son percibidos como más resistentes a las innovaciones (Leek et al., 2001, Tellis et al., 2009). En general la edad influye en la adopción de innovaciones, ya que los jóvenes son menos aversos al riesgo (Burgess y Steenkamp, 2006). En el mercado de alimentos, se ha observado esta tendencia en la adquisición de alimentos ecológicos (Bartels y Reinders, 2010; Rimal y Moon, 2005), alimentos funcionales (Krystallis et al., 2010; Leek et al., 2001), y alimentos étnicos (Pelchat y Pliner, 1995; Xu et al., 2004). De esta forma se plantea la primera de las hipótesis:

Hipótesis 1a: la edad del consumidor tiene un efecto negativo en el carácter innovador del consumidor

Por otro lado, la literatura señala que cuanto mayor es el estatus socioeconómico del consumidor y su nivel de estudios, mayor es la posibilidad de estar expuestos a distintos productos, de comer fuera del hogar, de interactuar con otras culturas y conocer otras cocinas, y por lo tanto es mayor la posibilidad de ser más innovador (Flight et al., 2003; Tuorila et al. 2001). Esto da lugar a las siguientes hipótesis:

Hipótesis $1 \mathrm{~b}$ : el nivel educativo del consumidor tiene un efecto positivo en el carácter innovador del consumidor
Hipótesis 1c: el nivel económico del consumidor tiene un efecto positivo en el carácter innovador del consumidor

Por último, tal y como ya se ha señalado con anterioridad, la mayor parte de los trabajos han mostrado el papel de la innovativeness o tendencia innovadora del consumidor como un elemento clave y positivo en la adopción de innovaciones (Banterle et al., 2011; Dobre et al., 2009; Goldsmith y Flynn, 1995; Roehrich, 2004), lo que motiva la siguiente hipótesis:

Hipótesis 2: el carácter innovador del consumidor tiene un efecto positivo en la adopción de innovaciones

Estas hipótesis dan lugar al modelo inicial, que se muestra en la Figura $1 a$.

\section{Efecto de las emociones}

El término emoción es usado para referirse a un estado mental de preparación que surge del aprendizaje cognitivo de eventos o pensamientos, acompañado normalmente por un proceso psicológico y que se suele expresar de forma física (gestos, posturas, etc.). Puede dar lugar a acciones específicas para afirmar la emoción, dependiendo de la naturaleza y el significado que para la persona tenga (Lazarus, 1991; Oatley, 1992). El estudio de las emociones se ha visto limitado y coartado por la ambigüedad entre dos cuestiones interrelacionadas: la estructura y el contenido de las emociones (Bagozzi y Yi, 1988). Con respecto a la estructura de las emociones, algunos investigadores (Izard, 1977) examinan todas las emociones al mismo nivel de generalidad, mientras que otros especifican una estructura jerárquica en las que las emociones específicas son casos particulares de una emoción más general (Shaver et al., 1987; Storm y Storm, 1987). En referencia al contenido de las emociones existe un debate: unos investigadores indican que sería más fructífero concebirlas como factores generales más amplios, como placer/excitación 


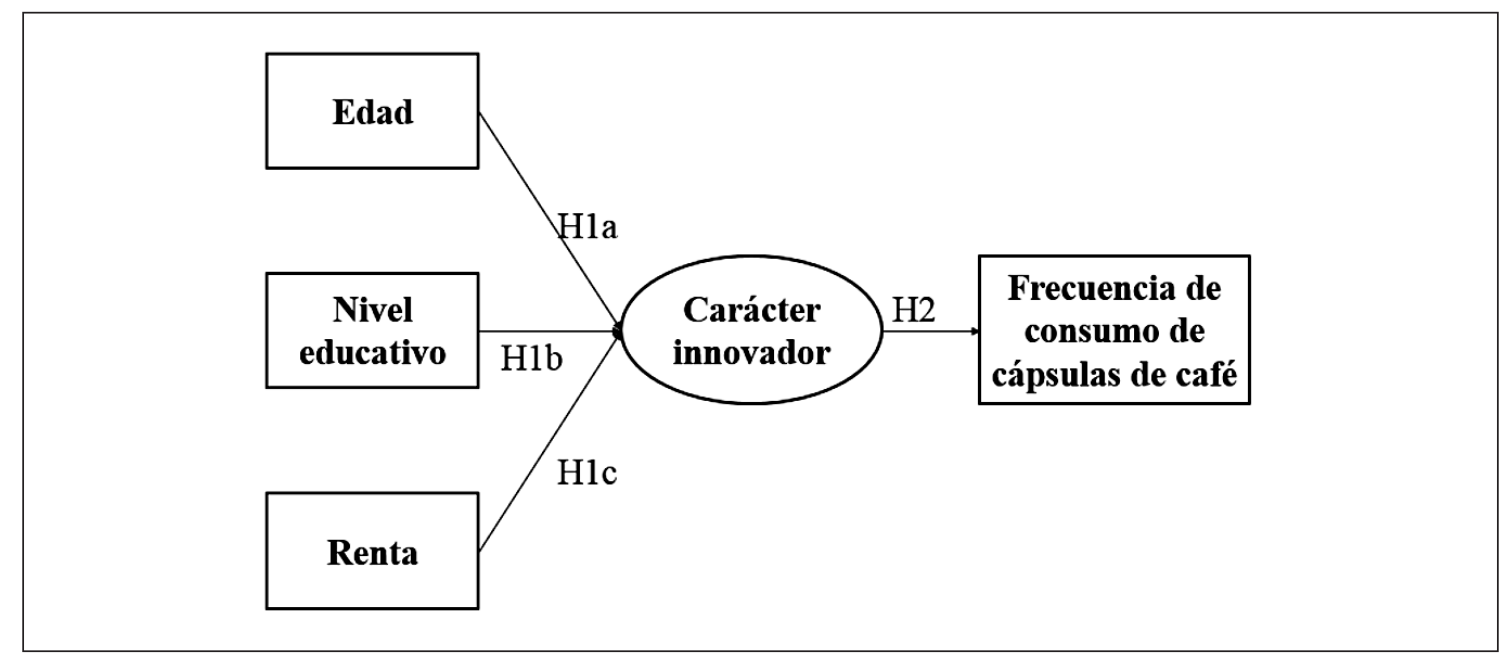

Figura 1a. Modelo inicial. Determinantes socioeconómicos del carácter innovador en la frecuencia de consumo de cápsulas de café.

Figure 1a. Initial model. Socioeconomic determinants of innovativeness in the frequency of coffee capsules' consumption.

(Russell, 1980) o a efectos positivos/negativos (Watson y Tellegen, 1985). Por otro lado, otros consideran que no hay que considerarlas en general porque cada emoción tiene sus propios estimadores (Frijda et al, 1989; Roseman et al., 1996).

Las emociones pueden afectar la elección de alimentos (Macht, 2008), incrementando la ingesta de unos o desechando otros. Además, autores como Narchi et al. (2008) recalcan la influencia de las emociones en el proceso de elección de alimentos por parte de los consumidores, en la percepción de calidad alimentaria y en los niveles de satisfacción indicados. Al mismo tiempo que señalan la falta de investigación en este campo.

La ingestión de alimentos viene controlada en gran medida por las emociones (Desmet y Schifferstein, 2008; Edwards et al., 2013; Evers et al., 2013, Hartwell et al, 2013; Kulviwat et al., 2007; Macht, 2008; Piqueras-Fiszman y Jaeger, 2014). Muchas personas comen para distraerse, para compensar emociones negativas como estrés, ansiedad, frustración, miedo, tristeza, aburrimiento, depresión, fatiga (Chua et al., 2004; O'Connor, et al., 2008; Polivy y Herman, 1999; Schachter et al., 1968; Wallis y Hetherington, 2004, 2009; Willner et al., 1998). En un estudio de Macht (1999) se encontraron mayores niveles de hambre durante momentos de enfado y disfrute que de miedo y envidia, dándose una tendencia a comer más cuando se experimentan emociones negativas. La mayor parte de los consumidores tienden a elegir alimentos que emocionalmente son cercanos a ellos (Jiang et al., 2014; Porcherot et al., 2010).

Del mismo modo, se sugiere una relación positiva entre las emociones positivas y el carácter creativo o innovador del consumidor (Fredrickson, 2003). Las emociones positivas como el amor, paz, optimismo, orgullo, alivio, alegría, excitación, dan lugar a la apertura de mente y por lo tanto tienen una influencia favorable en el carácter innovador del individuo (Amabile et al., 2005; Isen et al., 1987). Por ejemplo, la alegría y el disfrute dan lugar a 
una activación de la jocosidad (Frijda, 1986), que estimula el interés por la exploración y la creatividad (Fredrickson y Losada, 2005). Asimismo, la resistencia a las innovaciones y el carácter menos innovador están íntimamente relacionados con emociones negativas, como el enfado, miedo, tristeza, disgusto, culpa, vergüenza, envidia y preocupación (Bagozzi y Lee, 1999; Garg y Lerner, 2013). A partir de estas proposiciones se plantean las hipótesis:
Hipótesis 3a: las emociones positivas tienen un efecto significativo sobre el carácter innovador del consumidor.

Hipótesis 3b: las emociones negativas tienen un efecto significativo sobre el carácter innovador del consumidor.

La introducción de las emociones dan lugar al modelo ampliado, que se muestra en la Figura $1 b^{1}$.

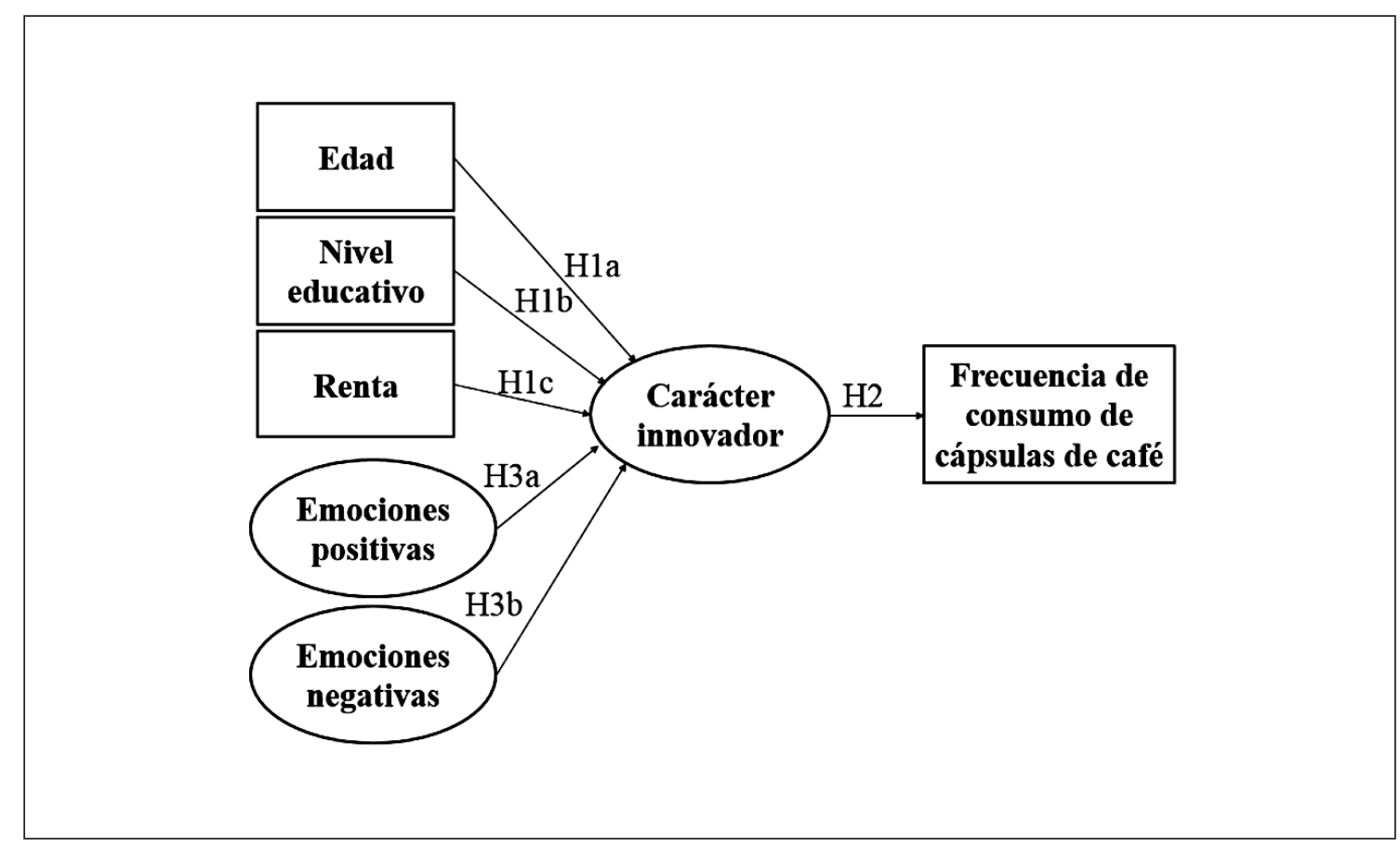

Figura 1b. Modelo ampliado. Determinantes socioeconómicos y emocionales del carácter innovador en la frecuencia de consumo de cápsulas de café. Figure $1 \mathrm{~b}$. Extended model. Socioeconomic and emotional determinants of innovativeness in the frequency of coffee capsules' consumption.

1. El modelo estructural propuesto se trata de un modelo formativo, donde tal y como sugiere Bollen (1989) las variables precursoras (edad, nivel educativo y renta) causan una variable latente (carácter innovador) la cual, a su vez, determina la frecuencia de consumo. 
Metodología

\section{Producto elegido}

Para la consecución del objetivo expuesto se ha seleccionado una innovación como es el café en cápsulas (tipo Nespresso), un sistema de consumo de café que ha revolucionado la forma de comprar y consumir el café en España. La elección de este producto ha venido motivada por ser el café un producto ampliamente conocido y consumido en España. De acuerdo con datos del Ministerio de Agricultura, Alimentación y Medio Ambiente de España (MAGRAMA), los niveles de consumo para el café tradicional son muy elevados, consumiendo de forma habitual este producto el $80 \%$ de la población. En concreto, en 2012 el consumo per capita ha ascendido a 1,53 kilos, observándose una tendencia mantenida en los últimos años según los datos del Panel de Consumo Alimentario del MAGRAMA. En el caso de las cápsulas de café, la frecuencia de consumo es mucho menor que para el producto tradicional. El despegue de esta innovación propuesta se produjo entre los años 2004-2005², habiendo triplicado su penetración en el mercado español en los dos últimos años, superando ya la barrera de los 1,5 millones de consumidores habituales, (lo que supone el $8 \%$ del volumen de consumo de café) (MAGRAMA, 2011). En 2010 se calculaba que el $35 \%$ de los hogares de más de una persona disponía ya de algún equipo que funcione específicamente con café en cápsulas. Por otro lado, la demanda creciente ha hecho que muchos tostadores de café tengan ya sistema propio de encapsulado.

\section{Obtención de información}

La información para conseguir los objetivos propuestos ha sido recogida a través de una en- cuesta personal realizada en Navarra durante marzo y abril de 2011, dirigida a compradores de alimentos para el hogar. Los encuestados respondían a diversas proposiciones para conocer sus emociones cuando consumen las cápsulas de café y se medía su espíritu innovador a través de la escala Domain Specific Innovativeness-DSI. En la encuesta también se recogieron las características sociodemográficas de los encuestados.

La escala de Domain Specific Innovativeness (DSI) (Goldsmith y Flynn, 1995) es una escala fiable y válida para medir el grado de innovación del consumidor dentro de una categoría específica de producto, lo que refleja la tendencia a adoptar o probar nuevos productos en un sector determinado tras su aparición en el mercado (Goldsmith y Hofacker, 1991).

Se ha trabajado con una muestra de conveniencia compuesta por 98 personas responsables de la compra de alimentos en el hogar, que acudieron a una convocatoria personal realizada por correo electrónico a personal (profesores, personal de Administración y Servicios y estudiantes) de la Universidad Pública de Navarra. Las características de la muestra y de la población de Navarra se exponen en la Tabla 1.

La mayor diferencia surge en relación con el mayor porcentaje de participantes con un alto nivel educativo, debido a que la encuesta fue desarrollada en la Universidad. También se aprecia un mayor número de mujeres en la muestra utilizada, debido probablemente a que la encuesta se realizó a personas responsables de la compra en el hogar, y todavía sigue habiendo un mayor número de mujeres que realiza esta función. Sin embargo, aunque se puede considerar que la muestra se encuentra sesgada en lo que a nivel educa-

2. El café encapsulado para máquinas comenzó a comercializarse en 1986, no obstante en España se trata de un producto innovador, ya que su introducción al mercado fue más tardía (Nesspreso, 2012). 
tivo respecta, lo cierto es que otros elementos como la composición del hogar, edad y género mantienen una tendencia y composición similar a la población de Navarra.

Tabla 1. Características de la muestra y de la población de Navarra

Table 1. Characteristics of the sample and the population of Navarra

\begin{tabular}{lcc}
\hline & Muestra & Población \\
\hline $\begin{array}{lcc}\text { Género, \% } \\
\text { Masculino }\end{array}$ & 28,03 & 49,77 \\
Femenino & 71,97 & 50,23 \\
Edad media & 40,03 & 40,50 \\
Tamaño del hogar & 3,06 & 2,90 \\
Nivel educativo, \% & & \\
$\quad$ Elemental & - & 18,67 \\
$\quad$ Medio & 17,99 & 52,24 \\
$\quad$ Superior & 82,10 & 29,09 \\
\hline
\end{tabular}

Fuente: Elaboración propia e Instituto Nacional de Estadística (INE, 2007).

\section{Análisis de los datos}

Este trabajo utiliza los programas SPSS Statistics 20.0 y AMOS 20.0 para analizar los datos bajo el algoritmo de máxima probabilidad. Siguiendo a Anderson y Gerbing (1988), en primer lugar se estima el modelos de medida usando el análisis factorial confirmatorio (AFC) para garantizar que los ítems preestablecidos para reflejar un mismo constructo latente están altamente correlacionados entre sí y, por lo tanto, son fiables. Además, se utiliza el coeficiente alfa de Cronbach (1951) para evaluar la consistencia interna de los ítems, donde alfa debe ser superior a 0,7 y la correlación entre los ítems debe exceder de 0,3 (Nunnally, 1978). La adecuación de cada escala multi-ítem en la captura de su respectivo constructo se examina a continuación para contrastar la validez convergente del modelo. Así, se evalúa la validez interna del modelo de medida mediante el cálculo de la fiabilidad compuesta, que debe tener un valor superior a 0,6 (Bagozzi et al., 1999), y a través de la varianza extraída, superior a 0,5 (Hair et al., 1998). Finalmente, se probó la validez discriminante para determinar si los constructos del modelo son diferentes entre sí. Fornell y Larcker (1981) recomiendan el uso de la varianza extraída. Así, sugieren que la varianza extraída debe ser superior que la varianza compartida entre el constructo con el resto de los constructos del modelo, esto es, la correlación al cuadrado entre dos constructos.

Después de evaluar la adecuación de los modelos de medida, se utilizan los modelos de ecuaciones estructurales para comprobar las relaciones causales. Como es habitual, los resultados de los modelos de ecuaciones estructurales se pueden interpretar en términos de importancia de los coeficientes individuales y en términos de bondad de ajuste (Jöreskog y Sörbom, 1988). En cuanto a la bondad del ajuste, por lo general se recomienda utilizar varios índices, como: chi-cuadrado $\left(\chi^{2}\right)$; la razón de chi-cuadrado sobre los grados de libertad $\left(\chi^{2} / \mathrm{gl}\right)$ que debe ser inferior a 3; y los índices CFI (índice de ajuste comparativo), GFI (índice de bondad de ajuste) e IFI (índice de ajuste incremental) que deberían ser próximos a 0,9 o 1,0 y RMSEA (error de aproximación cuadrático medio) que no debería exceder de 0,1 y lo ideal sería que estuviese entre 0,05 y 0,08 (Hair et al., 1998). Por último, para determinar si la inclusión de variables emocionales en el modelo final mejora la explicación del modelo inicial propuesto se va a emplear la medida $\mathrm{R}^{2}$ ajustado que refleja el porcentaje de varianza explicado de la variable dependiente (frecuencia de consumo) por los determinantes considerados en cada uno de los modelos. 


\section{Resultados}

Fiabilidad y validez del modelo

La lista completa de ítems incluyendo sus medias y desviaciones típicas aparecen en la Tabla 2. Así, se aprecia como el consumidor tipo encuestado tiene una media de 40 años, estudios superiores o universitarios y un poder adquisitivo medio $(1.500 €-3.000 €)$. En términos del carácter innovador del consumidor, destaca la compra ciega de nuevos productos frente a las otras características. Por otra parte, con respecto a las emociones positivas percibidas en el consumo de cápsulas de café sobresalen el optimismo, contento y la excitación. En la percepción de emociones negativas, destacan la impaciencia, la envidia y la preocupación. Finalmente, se aprecia un consumo actual de cápsulas de café relativamente bajo.

A continuación, se inicia la validación del modelo de medida. Así, se evalúa la consistencia interna de los ítems que componen cada escala usando el coeficiente alfa de Cronbach. Como resultado, se eliminó un ítem de la escala DSI ("aún cuando haya nuevos alimentos no los compro"), cuatro ítems de la escala emociones positivas ("amor romántico", "excitación", "orgullo", "sorpresa") y de la escala de emociones negativas ("cólera", "envidia", "vergüenza", "soledad"). Todos los ítems fueron eliminados con el propósito de mejorar la fiabilidad de los modelos. Una vez eliminados los ítems con una correlación inferior a 0,3, todas las escalas presentaron una fiabilidad superior a 0,7. Una vez analizada la fiabilidad de las escalas, se realizó un AFC para evaluar la estructura subyacente de las variables que integran el modelo. El AFC evalúa el modelo de medida para los constructos carácter innovador, emociones positivas y emociones negativas. Los resultados del AFC indican que el modelo propuesto se ajusta bien a los datos de la muestra $\left(\chi^{2}=165,10, P<0,01\right.$, RMSEA $=0,08$,
$\mathrm{CFI}=0,91, \mathrm{GFI}=0,85, \mathrm{IFI}=0,92$ ). Todos los coeficientes de regresión estandarizados de los ítems individuales con respecto a sus variables latentes fueron superiores al umbral de 0,4 . Además, todos los ítems se asociaron significativamente con respecto a su variable especifica $(p<0,01)$. Asimismo, todas las escalas han pasado con éxito las pruebas de fiabilidad compuesta $(>0,7)$ y varianza extraída (próxima o superior a 0,5 ), mostrando que la validez convergente del modelo de medida es adecuada ya que todas las escalas presentan una fiabilidad y validez de moderada a alta. Finalmente, la Tabla 3 confirma la validez discriminante del modelo propuesto y muestra como la mayoría de las escalas están correlacionadas entre sí $(P<0,10)$. Por lo tanto, se confirma la adecuación del modelo de medida propuesto a nuestros datos.

\section{Modelos estructurales}

Una vez garantizada la adecuación del modelo de medida, se procede con el análisis de los resultados obtenidos en los modelos estructurales correspondientes al modelo inicial (factores socioeconómicos, carácter innovador y frecuencia de consumo) y al modelo ampliado (factores socioeconómicos, factores emocionales, carácter innovador y frecuencia de consumo). El modelo inicial presenta un ajuste inaceptable debido al elevado error de medida $(>0,80): \chi^{2}=60,21(p<0,01)$, RMSEA $=$ $0,11, \mathrm{CFI}=0,86, \mathrm{GFI}=0,88, \mathrm{IFI}=0,87$. Además, sólo el carácter innovador muestra diferencias significativas con respecto al consumo de cápsulas de café $(\beta=0,22, \mathrm{P}<0,05)$. Por otra parte, no se ha podido confirmar el efecto de ninguna variable socioeconómica en el carácter innovador de los consumidores encuestados $(P>0,10)$. Los determinantes, edad, estudios y renta, explican el $4 \%$ de la varianza del carácter innovador y el carácter innovador refleja el $4 \%$ de la varianza de la frecuencia de consumo de café. 
Tabla 2. Fiabilidad y Análisis Factorial Confirmatorio

Table 2. Reliability and Confirmatory Factorial Analysis

\begin{tabular}{|c|c|c|c|c|}
\hline Variables & $\begin{array}{l}\text { Media (dt) / } \\
\text { Frecuencia }\end{array}$ & $\beta$ & AVE & $\mathrm{FC}$ \\
\hline Renta, \% & & - & & \\
\hline Baja $(>1500 €)$ & 13,3 & & & \\
\hline Media $(1500 €-3000 €)$ & 58,2 & & & \\
\hline Alta $(>3000 €)$ & 28,6 & & & \\
\hline Carácter innovador $(\alpha=0.78)$ & & & 0,51 & 0,82 \\
\hline Compro nuevos alimentos antes que la mayoría de la gente & $2,01(0,96)$ & 0,79 & & \\
\hline Soy el primero de mi círculo en comprar nuevos alimentos & $2,10(1,04)$ & 0,87 & & \\
\hline Comparado con mi entorno, compró más nuevos alimentos & $2,25(0,99)$ & 0,94 & & \\
\hline Aún cuando haya nuevos alimentos en la tienda, no los compro & $2,25(1,05)$ & ni & & \\
\hline Soy el último de mi círculo en conocer las nuevas tendencias & $2,15(1,09)$ & 0,34 & & \\
\hline Compraría un nuevo alimento aún sin haberlo probado & $3,05(1,22)$ & 0,37 & & \\
\hline Emociones positivas $(\alpha=0.81$ ) & & & 0,39 & 0,79 \\
\hline Amor romántico & $1,63(0,95)$ & ni & & \\
\hline Paz & $2,68(1,35)$ & 0,76 & & \\
\hline Optimismo & $2,91(1,31)$ & 0,75 & & \\
\hline Excitación & $2,77(1,29)$ & ni & & \\
\hline Orgullo & $1,31(0,78)$ & ni & & \\
\hline Alivio, consuelo & $2,13(1,21)$ & 0,64 & & \\
\hline Amor & $1,57(0,89)$ & 0,49 & & \\
\hline Contento & $2,83(1,29)$ & 0,52 & & \\
\hline Alegría, júbilo & $2,44(1,13)$ & 0,53 & & \\
\hline Sorpresa & $1,22(0,63)$ & ni & & \\
\hline Emociones negativas $(\alpha=0.74)$ & & & 0,38 & 0,78 \\
\hline Cólera, ira & $1,03(0,23)$ & $\mathrm{ni}$ & & \\
\hline Preocupación & $1,25(0,69)$ & 0,79 & & \\
\hline Miedo & $1,08(0,42)$ & 0,82 & & \\
\hline Envidia & $1,63(0,94)$ & $\mathrm{ni}$ & & \\
\hline Descontento & $1,07(0,26)$ & 0,54 & & \\
\hline Tristeza & $1,13(0,49)$ & 0,56 & & \\
\hline Vergüenza & $1,04(0,24)$ & ni & & \\
\hline Soledad & $1,24(0,62)$ & ni & & \\
\hline Impaciencia & $1,66(1,05)$ & 0,49 & & \\
\hline Culpa & $1,12(0,54)$ & 0,39 & & \\
\hline Frecuencia de consumo de cápsulas de café & $1,62(0,81)$ & - & & \\
\hline
\end{tabular}

ß: ponderación estandarizada; a fiabilidad (a de Cronbach); FC: fiabilidad compuesta; AVE: varianza extraída; ni: ítem no incluido en el modelo de medida por no superar la validación inicial. 
Tabla 3. Varianza extraída (AVE) y correlaciones entre los constructos latentes Table 3. Correlations in latent constructs

\begin{tabular}{lcccc}
\hline & & \multicolumn{3}{c}{ Correlaciones } \\
\cline { 3 - 5 } & AVE & $\begin{array}{c}\text { Carácter } \\
\text { innovador }\end{array}$ & $\begin{array}{c}\text { Emociones } \\
\text { positivas }\end{array}$ & $\begin{array}{c}\text { Emociones } \\
\text { negativas }\end{array}$ \\
\hline Carácter innovador & 0,51 & & & \\
Emociones positivas & 0,39 & $0,19 *$ & & \\
Emociones negativas & 0,38 & $-0,02$ & $0,21^{*}$ & \\
\hline
\end{tabular}

${ }^{*} p<0,10$.

A continuación, se prueba el modelo ampliado que muestra un ajuste aceptable en términos de los índices utilizados: $\chi^{2}=271,85$ $(p<0,01)$, RMSEA $=0,07, \mathrm{CFI}=0,87, \mathrm{GFI}=$ $0,81, \mathrm{IFI}=0,87$. Los resultados muestran que el nivel educativo $(\beta=0,17, P<0,10)$ y la percepción de emociones positivas $(\beta=0,22$, $\mathrm{P}<0,05)$ influyen positivamente en el carácter innovador de los consumidores. Por su parte, el carácter innovador determina significativamente la frecuencia de consumo de cápsulas de café $(\beta=0,23, P<0,05)$. Por tanto, se confirma que dos aspectos importantes que favorecen que los consumidores sean más proclives a innovar en el consumo de café son una mayor formación y los productos que evocan emociones positivas. Además, un fuerte carácter innovador desencadena un mayor consumo de las nuevas cápsulas de café. Con respecto a las varianzas explicadas, los determinantes edad, estudios, renta, emociones positivas y emociones negativas explican el $9 \%$ de la varianza del carácter innovador y el carácter innovador cubre $5 \%$ de la frecuencia de consumo de cápsulas de café.

\section{Comparación de modelos}

Sólo el modelo ampliado mostró un ajuste aceptable. Esto implica que sólo el modelo ampliado podría predecir adecuadamente la frecuencia de consumo de cápsulas de café. Además, los resultados indican que el modelo ampliado tiene un mejor poder explicativo para el carácter innovador $\left(R^{2}\right.$ ajustada $=$ $0,09)$ y para la frecuencia de consumo $\left(R^{2}\right.$ ajustada $=0,05)$ que el modelo inicial $\left(R^{2}\right.$ ajustada $=0,04$ en ambos casos). Aunque, algunos de sus índices de ajuste son ligeramente inferiores que el modelo inicial (GFI = 0,81 ), la mayoría de los índices de comparación del modelo ampliado muestran un ajuste superior $\left(\chi^{2} / \mathrm{df}=1,47, \mathrm{CFI}=0,87\right.$, RMSEA $=0,07)$. Se puede concluir que el modelo ampliado identifica la frecuencia de consumo y de sus determinantes más próximos con mayor detalle que el modelo inicial, aunque aún existe un amplio porcentaje de varianza sin explicar.

\section{Discusión}

A pesar de los esfuerzos realizados por la industria agroalimentaria el índice de fracaso de los nuevos alimentos en su salida al mercado es muy elevado, debido entre otras razones, a una falta de entendimiento del consumidor. Por eso se muestra muy necesario conocer al consumidor, descubrir qué busca en los productos y qué aspectos de su perso- 
nalidad está proyectando a través de los alimentos que busca para su consumo. Tradicionalmente el estudio del comportamiento del consumidor en relación con innovaciones se ha centrado en características demográficas asociadas con la disposición a adoptar nuevos productos, pero estudios más recientes incorporan la innovativeness o tendencia innovadora del consumidor como un factor positivo en la adopción de innovaciones, y la influencia que tienen las emociones percibidas respecto al nuevo producto.

Este estudio piloto trata de analizar en qué medida el carácter innovador del consumidor determina el consumo de nuevos alimentos, y cómo las emociones percibidas ayudan a mejorar la predicción del consumo de nuevos alimentos.

Para ello se han utilizado los modelos de ecuaciones estructurales y se han comparado dos modelos: un modelo inicial en el que se consideran los factores socioeconómicos, el carácter innovador y la frecuencia de consumo, y un modelo ampliado donde se incorporan además, las emociones percibidas por parte del consumidor al consumir el nuevo alimento.

Los resultados obtenidos con el modelo ampliado, indican que el nivel educativo y las emociones positivas influyen positivamente en el carácter innovador de los consumidores y este último determina significativamente la frecuencia de consumo de cápsulas de café. Estos resultados están la línea de los obtenidos en investigaciones previas donde ya se ha demostrado que los consumidores de mayor nivel educativo son más proclives a probar nuevos productos (Flight et al., 2003; Tuorila et al., 2001). Por su parte, las emociones percibidas en el consumo de nuevos alimentos se han configurado como el predictor más significativo del carácter innovador de los consumidores (Evers et al., 2010; Köster, 2009). Finalmente, también se ha confirmado que los consumidores más predispuestos a probar nuevos alimentos son los que poseen un comportamiento de adopción más innovador (Banterle et al., 2011, Dobre et al., 2009).

Como conclusión podemos señalar que hay dos aspectos importantes que favorecen que los consumidores sean más proclives a innovar en el consumo de café: el grado de formación académica y la evocación de emociones positivas. Ayudar a los consumidores a reconocer como las emociones positivas o negativas influyen en su relación con la comida puede asistirlos a la hora de hacer cambios positivos en su dieta. La inclusión de palabras relacionadas con emociones positivas en las etiquetas, pueden provocar respuestas positivas en el consumo. Se ha comprobado además que, un fuerte carácter innovador implica un mayor consumo de cápsulas de café.

Puesto que las cápsulas de café son un producto en expansión parece que la evocación de emociones positivas unidas a la imagen del producto es muy interesante para su introducción en segmentos de consumidores más tardíos en la incorporación de innovaciones e incluso para aumentar el grado de consumo de los que ya lo consumen.

Por último, y teniendo en cuenta que se trata de un estudio piloto, debemos mencionar como limitación, que al centrarse en el café en cápsulas, no se pueden generalizar los resultados a otros nuevos alimentos. Asimismo, se ha detectado que existe todavía un amplio porcentaje de varianza sin explicar en la frecuencia de consumo de café. Por esta razón, sería conveniente considerar otros factores demográficos (género, lugar de residencia) o psicológicos (satisfacción, actitudes, normas subjetivas) que ayudasen a mejorar la explicación del modelo. Por ello, resultaría interesante ampliar el alcance de la investigación a otras innovaciones alimentarias o bien a otros mercados, y en cualquier caso a muestras más grandes de consumidores. 


\section{Agradecimientos}

Los autores agradecen la financiación recibida a través del proyecto de investigación AGL 2012-39793-C03-01.

\section{Bibliografía}

Amabile TM, Barsade SG, Mueller JS, Staw BM (2005). Affect and creativity at work. Administrative Science Quarterly 50(3): 367-403.

Anderson JC, Gerbing DW (1988). Structural Equation Modeling in Practice: A Review and Recommended Two-Step Approach. Psychological Bulletin Copyright 1988 by the American Psychological Association 103: 41-423.

Bagozzi R, Gopinath M, Nyer P (1999). The role of emotions in marketing. Journal Academy of Marketing Science 27: 184-206.

Bagozzi R, Yi Y (1988). On the evaluation of structural equations models. Journal of Academy of Marketing Science 16: 74-94.

Bagozzi RP, Lee K-H (1999). Consumer resistance to and acceptance of innovations. Advances in consumer Research 26: 218-225.

Banterle A, Cavaliere A, Carraresi L, Stranıeri S (2011). Innovativeness in food small business: What is its relationship with marketing?. Agricultural Economics - Czech 57: 474-483.

Baregheh A, Rowley J, Sambrook S (2009). Towards a multidisciplinery definition of innovation. Journal of Management Decision 47(8): 1323-1339.

Bartels J, Reinders MJ (2010). Social identification, social representations and consumer innovativeness in an organic food context: a cross-national comparison. Food quality and Preference 21: 347-352.

Bellows AC, Alcaraz G, Hallman WK (2010). Gender and food, a study of attitudes in the USA towards organic, local, U.S. grown, and GMfree foods. Appetite 55: 540-550.

Bollen K (1989). Structural equations with latent variables. John Wiley \& Sons, Nueva York, EE. UU.
Burgess SM, Steenkamp J-BEM (2006). Marketing renaissance: How research in emerging markets advances marketing science and practice. International. Journal of Research in Marketing 23: 337-356.

Capitanio F, Coppola A, Pascucci S (2009). Indications for drivers of innovation in the food sector. British Food Journal 111(8): 820-838.

Chua JL, Touyz S, Hill AJ (2004). Negative mood-induced overeating in obese binge eaters: An experimental study. International Journal of Obesity 28, 606-610.

Costa AIA, Dekker M, Jongen WMF (2004). An overview of means-end theory: potential application in consumer-oriented food product design. Trends in Food Science and Technology 15: 403-415.

Cronbach LJ (1951). Coefficient alpha and the internal structure of tests. Psychometrika 16, 297334.

Desmet PMA, Schifferstein HNJ (2008). Sources of positive and negative emotions in food experience. Appetite 50: 290-301.

Dobre C, Dragomır A, Preda G (2009). Consumer Innovatıveness: A Marketıng Approach. Management \& Marketing 4(2):19-34.

Edwards JSA, Hartwell HJ, Brown L (2013). The relationship between emotions, food consumption and meal acceptability when eating out of the home. Food Quality and Preference 30(1): 22-32.

Evers C, Adriaanse M, de Ridder DTD, deWitt Huberts JC (2013). Good mood food. Positive emotion as a neglected trigger for food intake. Appetite 68(1): 1-7.

Evers C, Stok FM, Ridder DTD (2010). Feeding Your Feelings: Emotion Regulation Strategies and Emotional Eating. Personality and Social Psychology Bulletin 36(6): 792-804.

Flight I, Leppard P, Cox DN (2003). Food neophobia and associations with cultural diversity and socio-economic status amongst rural and urban Australian adolescents. Appetite 41: 51-59.

Fornell C, Larcker DF (1981). Evaluating Structural Equation Models with Unobservable Variables and Measurement Error. Journal of Marketing Research18(1): 39-50. 
Fortuin FTJM, Omta SWF (2009). Innovation drivers and barriers in food processing. British Food Journal 111(8): 839-851.

Foxall GR (1988). Consumer Innovativeness: Novelty Seeking, Creativity, and Cognitive Style En: Research in Consumer Behavior. Vol. 3 (Eds, EC Hirschman y JN Sheth) Greenwich, CT: JAI Press, pp. 79-113.

Foxall GR (1995). Cognitive styles of consumer initiators. Technovation 15(5): 269-288.

Fredrickson BL (2003). The value of positive emotions: The emerging science of positive psychology is coming to understand why it's good to feel good. American Scientist 91: 330-335.

Fredrickson BL, Losada MF (2005). Positive affect and the complex dynamics of human flourishing. American Psychologist 60(7): 678-686.

Frijda NH (1986). The emotions. Cambridge: Cambridge University Press.

Frijda NH, Kuipers P, Schure E (1989). Relations among emotion, appraisal, and emotional action readiness. Journal of Personality and Social Psychology 57(2): 212-228.

Garg N, Lerner JS (2013). Sadness and consumption. Journal of Consumer Psychology 23(1): 106-113.

Gatignon H, Robertson TS (1991). Innovative decision processes. En: Consumer Behavior. (Eds. T.S. Robertson, y H.H. Kassarjian (eds). 316-346. Englewood Cliffs, NJ: Prentice-Hall.

Goldsmith RE, d'Hauteville F, Flynn LR (1998). Theory and measurement of consumer innovativeness. A transnational evaluation. European Journal of Marketing. 32: 340-353.

Goldsmith RE, Flynn LR (1995). The domain specific innovativeness scale: theoretical and practical dimensions. Association for Marketing Theory and Practice Proceedings 4: 177-82.

Goldsmith RE, Hofacker CF (1991). Measuring consumer innovativeness. Journal of the Academy of Marketing Science 19(3): 209-221.

Gourville JT (2006). Eager Sellers and Stony Buyers: Understanding the Psychology of New-Product Adoption. Harvard Business Review 84: 98-106.
Gresham G, Hafer J, Markowski E (2006). Interfunctional market orientation between marketing departments and technical departments in the management of the new product development process. Journal of Behavioral and Applied Management 8(1): 53-65.

Hair JF, Anderson RE, Tatham RL, Black WC (1998). Multivariate data analysis ( $5^{a}$ th ed.), Upper Saddle River, NJ: Prentice Hall.

Hartwell HJ, Edwards JS, Brown L (2013). The relationship between emotions and food consumption (macronutrient) in a foodservice college setting-A preliminary study. International Journal of Food Sciences and Nutrition 64: 261-268.

Hirschman EC (1980). Innovativeness, novelty seeking, and consumer creativity. Journal of Consumer Research 7(3): 283-295.

Isen AM, Daubmann KA, Nowicky GP (1987). Positive affect facilitates creative problem solving. Journal of Personality and Social Psychology 52: 1122-1131.

Izard C (1977). Human emotions. New York: Plenum Press.

Jiang Y, King JM, Prinyawiwatkul W (2014). A review of measurement and relationships between food, eating behavior and emotion. Trends in Food Science and Technology 36: 15-28.

Jöreskog KG, Sörbom D (1988). PRELIS: A program for multivariate data screning anda data summarization. A preprocessor for LISREL. Chicago, SSI.

Köster EP (2009). Diversity in the determinants of food choice: A psychological perspective. Food Quality and Preference 20: 70-82.

Krystallis A, Linardakis M, Mamalis S (2010). Usefulness of the Discrete Choice Methodology for Marketing Decision-making in New Product Development: An Example from the European Functional Foods Market. Agribusiness 26(1): 100-121.

Kühne B, Vanhonacker F, Gellynck X, Verbeke W (2010). Innovation in traditional food products in Europe: Do sector innovation activities match consumers' acceptance? Food Quality and Preference 21: 629-638. 
Kulviwat S, Bruner II GC, Kumar A, Nasco SA, Clark $\mathrm{T}$ (2007). Toward a unified theory of consumer acceptance of technology. Psychology and Marketing 24: 1059-1084.

Lancaster KJ (1966). A new approach to consumer theory. Journal of Political Economy 74: 132-157.

Laverie DA, Kleine RF, Xhultz S (1993). Linking emotions and values in consumption experiences: An exploratory study. Advances in Consumer Research. 20: 70-75.

Lazarus R (1991). Emotion and adaptation. New York: Oxford University Press.

Leek S, Szmigin I, Carrigan M (2001). Older consumers and food innovation. Journal of International Food and Agribusiness Marketing 12 (1): 71-89.

Macht M (1999). Characteristics of eating in anger, fear, sadness and joy. Appetite 33: 129-139.

Macht M (2008). How emotions affect eating: A five-way model. Appetite 50: 1-11.

MAGRAMA (2011). La alimentación en España. Disponible en: http://www.magrama.es

Manning KC, Bearden WO, Madden TJ (1995). Consumer innovativeness and the adoption process. Journal of Consumer Psychology 4(4): 329-345.

Michon C, O'Sullivan MG, Sheehan E, Delahunty CM, Kerry JP (2010). Study on the influence of age, gender and familiarity with the product on the acceptance of vegetal soups. Food Quality and Preference 21: 478-488.

Naidoo V (2010). Firm survival through a crisis: The influence of market orientation, marketing innovation and business strategy. Industrial Marketing Management 39(8): 1311-1320.

Narchi I, Walrand S, Boirie Y, Rousset S (2008). Emotions generated by food in elderly French people. Journal of Nutrition, Health and Aging 12(9): 626-33.

Nunnally JC (1978). Psychometric theory. New York: McGraw-Hill.

Oatley K (1992). Best laid schemes: The psychology of emotions. Cambridge: Cambridge University Press.
O'Connor DB, Jones F, Conner M, McMillan B, Ferguson $E$ (2008). Effects of daily hassles and eating style on eating behavior. Health Psychology 27: S20-\$31.

Pelchat ML, Pliner P (1995). Try it. You'll like it. Effects of information on willingness to try novel foods. Appetite 24: 153-165.

Piqueras-Fiszman B, Jaeger SR (2014). The impact of the means of context evocation on consumers' emotion associations towards eating occasions. Food quality and preference 37: 61-70.

Polivy J, Herman CP (1999). Distress and eating: why do dieters overeat? International Journal of Eating Disorders 26: 153-164.

Porcherot C, Delplanque S, Raviot-Derrien S, Calve BL, Chrea C, Gaudreau N (2010). How do you feel when you smell this? Optimization of a verbal measurement of odor-elicited emotions. Food Quality and Preference 21: 938-947.

Rimal A, Moon W (2005). Perceived risks of agrobiotechnology and organic food purchase in the United States Southern Agricultural Economics Association Annual Meeting. 5-9 febrero 2005, Little Rock Arkansas, EE. UU.,1-26.

Roehrich G (2004). Consumer innovativeness: Concepts and measurements. Journal of Business Research 57: 671-677.

Rogers EM (1962). Diffusion of Innovations. 1st edition. The Free Press. Nueva York, EE. UU.

Rogers EM (2003). Diffusion of innovation. 5th edition. The Free Press. Nueva York, EE. UU.

Roseman IJ, Antoniou AA, Jose PJ (1996). Appraisal determinants of emotions: Constructing a more accurate and comprehensive theory. Cognitive Emotions 10: 241-277.

Russell JA (1980). A Circumplex Model of Affect. Journal of Personality and Social Psychology 39 (6): 1161-1178.

Schachter S, Goldman R, Gordon A (1968). Effects of fear, food deprivation, and obesity on eating. Journal of Personality and Social Psychology 10: 91-97.

Shaver Ph, Schwartz J, Kirson D, O’Conner C (1987). Emotion knowledge: further exploration of a prototype approach. Journal of Personality and Social Psychology 52: 1061-86. 
Steenkamp J-BEM, Hofstede F, Wedel M (1999). A cross-national investigation into the individual and national cultural antecedents of consumer innovativeness. Journal of Marketing 63: 55-69.

Storm C, Storm T (1987). A taxonomic study of the vocabulary of emotions. Journal of Personal and Social Psychology 53: 805-816.

Tellis GJ, Yin E, Bell S (2009). Global consumer innovativeness: cross-country differences and demographic commonalities. Journal of International Marketing 17(2): 1-22.

Tuorila H, Lähteenmäki L, Pohjalainen L, Lotti L (2001). Food neophobia among the Finns and related responses to familiar and unfamiliar foods. Food Quality and Preference12: 29-37.

Urala N, Lähteenmäki L (2007). Consumers' changing attitudes towars functional foods. Food Quality and Preference 18: 1-12.

Venkatraman MP, Price LL (1990). Differentiating between cognitive and sensory innovativeness: concepts, measurement, and implications. Journal of Business Research 20(4): 293-315.
Wallis DJ, Hetherington MM (2004). Stress and eating: The effects of ego-threat and cognitive demand on food intake in restrained and emotional eaters. Appetite 43: 39-46.

Wallis DJ, Hetherington MM (2009). Emotions and eating: Self-reported and experimentally induced changes in food intake under stress. Appetite 52: 355-362.

Watson D, Tellegen A (1985). Toward a consensual structure of mood. Psychological Bulletin 98: 219-235.

Willner P, Benton D, Brown E, Cheeta S, Davies G, Morgan J (1998). Depression increases craving for sweet rewards in animal and human models of depression and craving. Psychopharmacology 136: 272-283.

Xu J, Shim,S, Lotz S, Almeida D (2004). Ethnic identity, socialization factors and culture-specific consumption behavior. Psychology and Marketing 21(2): 93-112.

(Aceptado para publicación el 10 de abril de 2015) 